\title{
SPEAKER RECOGNITION USING A KIND OF NOVEL PHONOTACTIC INFORMATION
}

\author{
Xiang Zhang, Xiang Xiao, Haipeng Wang, Hongbin Suo, Qingwei Zhao, Yonghong Yan \\ ThinkIT Speech Lab, Institute of Acoustics, Chinese Academy of Sciences, Beijing \\ \{xzhang, xxiao, wanghaipeng, hsuo, qzhao, yonghong.yan\}@ @ccl.ioa.ac.cn
}

\begin{abstract}
In this paper, we present a new modeling approach for speaker recognition, which uses a kind of novel phonotactic information as the feature for SVM modeling. Gaussian mixture models (GMMs) have been proven extremely successful for textindependent speaker recognition. The GMM universal background model (UBM) is a speaker-independent model, each component of which can be considered to be modeling some underlying phonetic sounds. Thus, the UBM can be regarded to characterize a speaker-independent voice. We assume that the utterances from different speakers should get different average posterior probabilities on the same Gaussian component of the UBM, and the supervector composed of the average posterior probabilities on all components of the UBM for each utterance should be discriminative. We use these supervectors as the features for SVM based speaker recognition. Experiment results show that the proposed approach demonstrates comparable performance with the state-of-the-art systems on NIST 2006 SRE corpus. Fusion results are also presented.
\end{abstract}

Index Terms - Speaker recognition, gaussian mixture model, universal background model, support vector machine

\section{INTRODUC'TION}

Currently, the dominant features used in speaker recognition systems are cepstral features extracted over short time spans. The modeling of these features is typically carried out in terms of log-likelihood ratio of Gaussian mixture models (GMMs), or discriminatively using support vector machines (SVMs). Gaussian mixture modeling-universal background model (GMM-UBM) [1] [2] and Gaussian mean supervectors-SVM (GSV-SVM) [2] [3] [4] are two basic and commonly used approaches for speaker recognition. The fusion of this two systems show excellent performance in task of speaker recognition. However, both of the two approaches use only short-term cepstral features. They ignore high-level information, such as the particular word usage or the acoustic variability of phonetic events when comparing different speakers.

This work is partially supported by MOST (973 program, 2004CB318106), the National Natural Science Foundation of China (10574140, 60535030), the National High Technology Research and Development Program of China (863 program, 2006AA01010, 2006AA01Z195).
Phone-conditioned [5] and word-specific [6], [7] cepstral models are a direct attempt to make models invariant to the choice of words. [5] utilized a speech recognition front-end to hypothesize the phonetic content of the utterance and then phone dependent models were refined in place of a global GMM. [6] focused on using word units from a speech recognizer as acoustic unit, and aimed to compare the same acoustic unit as spoken by different speakers. In [7], a textconstrained system designed to employ select high-frequency key-words in the speech stream is presented. However, these approaches have the drawback of fragmenting the speech data and require sufficiently accurate speech recognition.

In this paper, we present a new approach for speaker recognition which uses a kind of phonotactic information for modeling. The Gaussian components of the UBM can be considered to be modeling some underlying phonetic sound classes [8]. We assume that the utterances from different speakers should get different average posterior probability on the same Gaussian component. This reflects a fact that the pronunciation of the same sound unit may differ from one speaker to another. Thus, we concatenate these average posterior probabilities on all components of the UBM into a vector. This vector can be seen as a histogram describing the characteristic of the speaker's pronunciation. Different person would exhibit different patterns of histogram. Together with the SVM modeling technique, this phonotactic information is used as the feature for discriminative classification. This system is quite less computational complex than the traditional phonotacitic systems based on speech recognition.

This paper is organized as following: In section 2, we give a simple review of two basic approaches for speaker recognition which our proposed approach would be compared to: GMM-UBM and GSV-SVM. In section 3, our proposed system is presented in detail. Section 4 gives out the evaluation corpus and experiment results. Finally, we conclude in section 5.

\section{BASELINE SYSTEMS}

This section briefly reviews two basic state-of-the-art speaker recognition systems, which serve as the baseline systems in this work. The first baseline system is a GMM-UBM [1], in which speaker models are trained by adapting from a UBM 
using maximum a posteriori (MAP) adaptation [9]. Only means of Gaussian components are adapted.

The second baseline system is GMM mean supervectors system followed by support vector machines (GSV-SVM). GSV-SVM is based on GMMs which differ only in means, so the means of each GMM are classified by SVMs. The feature extraction and UBM training is done in the same way as GMM-UBM system. In this type of system, each training, testing and background utterance is represented by the means of its Gaussian components. The mean vectors of all mixture components are then concatenated to form one supervector for each utterance. (We normalized each mean by the corresponding standard deviation) [10].

\section{PROPOSED SYSTEM}

\subsection{Phonotactic Feature Extraction and SVM Modeling}

In the GMM-UBM speaker recognition system, the UBM is a weighted linear combination of $M$ Gaussian component densities. For a $D$-dimensional feature vector, $x$, the formula of the UBM is defined as

$$
p\left(x \mid \lambda_{U B M}\right)=\sum_{i=1}^{M} w_{i} p_{i}(x)
$$

where, $p_{i}(x), i=1, \ldots, M$, are the component densities and $w_{i}, i=1, \ldots, M$ are the mixture weights. Each component density is a $D$-variate Gaussian function of the form

$$
p_{i}(x)=\frac{1}{(2 \pi)^{D / 2}} \exp \left\{-\frac{1}{2}\left(x-\mu_{i}\right)^{T} \Sigma_{i}^{-1}\left(x-\mu_{i}\right)\right\}
$$

with mean vector $\mu_{i}$, and covariance matrix $\Sigma_{i}$. The mixture weights satisfy the constraint that $\sum_{i=1}^{M} w_{i}=1$. Thus, the UBM model can be denoted as

$$
\lambda_{U B M}=\left\{w_{i}, \mu_{i}, \Sigma_{i}\right\}, i=1, \ldots, M
$$

The UBM is a speaker-independent background model, and each Gaussian component of the UBM can be considered to be modeling a kind of underlying broad phonetic sounds. Thus, all the Gaussian components of the UBM can be considered to characterize a speaker-independent voice. Given some feature vectors from the hypothesized speaker, $X=$ $\left\{x_{1}, x_{2}, \ldots, x_{T}\right\}$, we first calculate the probabilistic alignment of the vectors into the UBM mixture components. That is, for mixture $i$ in the UBM, the posterior probability is computed as following:

$$
\operatorname{Pr}\left(i \mid x_{t}\right)=\frac{w_{i} p_{i}\left(x_{t}\right)}{\sum_{i=1}^{M} w_{j} p_{j}\left(x_{t}\right)}
$$

The posterior probability is the normalized likelihood ratio, so it can be seen as a kind of similarity. The larger the posterior probability is, the better the mixture can be used to represent that feature vector. Thus the average posterior probability for mixture $i$ can be well represent the similarity between the hypothesized speaker's voice and the speakerindependent voice. The average posterior probability for mixture $i$ is calculated as following:

$$
b_{i}=\frac{1}{T} \Sigma_{t=1}^{T} \operatorname{Pr}\left(i \mid x_{t}\right)
$$

Therefore, when given a hypothesized speaker's one utterance, all the posterior probabilities for the Gaussian components of the UBM can be considered as the voicecharacteristic-discrepancy in the form of a probabilistic vector between the hypothesized speaker and the universal speaker (modeled by the speaker-independent model). These posterior probabilities are concatenated into a supervector:

$$
\mathbf{b}=\left[b_{1}, b_{2}, \ldots, b_{M}\right]^{T}
$$

The elements of this supervector can be seen as a histogram describing the characteristic of speech sound units for a hypothesized speaker's speech utterance. The supervectors from the same speaker are always similar, and different speakers would exhibit different patterns of histogram. This fact can be illustrated by Figure 1. The utterances used are from NIST 2006 SRE corpus. The top two pictures are calculated using two different utterances from the same speaker, and the below ones are from another speaker. We can see that histograms from the same speaker are more similar than from different speakers.
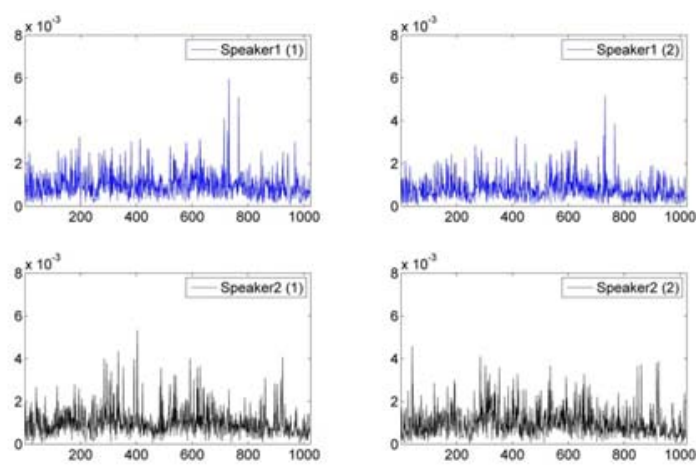

Fig. 1. Comparison of two speakers' phonotactic supervectors

Thus, this phonotactic information can be used as the feature for SVM based speaker recognition. The procedure for extracting the phonotactic features is shown in Figure 2.

Furthermore, the proposed algorithm is quite less computational complex than the traditional system based on phoneconditioned or word-specific models, and it is also faster than the GMM-UBM and GMM-SVM systems. 


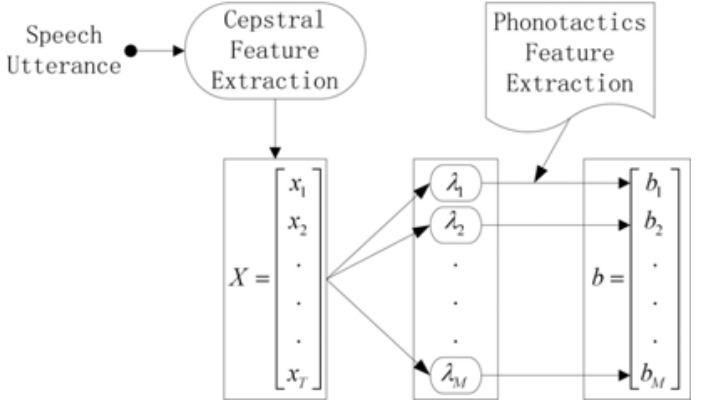

Fig. 2. The procedure of the phonotactic feature extraction

\subsection{Further Improvement}

When the large UBM is evaluated for a feature vector, only a few of the mixtures contribute significantly to the posterior value. This is because the UBM represents a distribution over a large space but a single cepstral feature vector will be near only a few components of the UBM. Thus, only the top $C$ largest scoring mixture components are used for calculating the average posterior values, where $C$ is an empirical value.

\section{EXPERIMENTS}

\subsection{Experimental Setup}

We performed experiments on the 2006 NIST speaker recognition (SRE) corpus. We focused on the single-side 1 conversation train, single-side 1 conversation test, and the multilanguage handheld telephone task (the core test condition) [11]. This setup resulted in 3,612 true trials and 47,836 false trials. We used equal error rate (EER) and the minimum decision cost value (minDCF) as metrics for evaluation [11].

For cepstral feature extraction, a $20 \mathrm{~ms}$ Hamming window with $10 \mathrm{~ms}$ shifts is used. Each utterance is converted into a sequence of 36-dimensional feature vectors, each consisting of 12 MFCC coefficients and their first and second derivatives. An energy-based speech detector is applied to discard vectors from low-energy frames. To mitigate channel effects, feature warping, cepstral mean subtraction and variance normalization are applied to the features.

The GMM UBM consists of 2048 mixture components. For GMM MAP training, we adapt only the means with a relevance factor of 12. The GMM UBM is trained using EM with the data from the corpora: NIST 01, NIST 02, NIST 04 and NIST 05. These data do not occur in any of the test sets. All systems in our experiments use the same UBM.

In both GSV-SVM and the proposed system, SVMTorch [12] with a linear inner-product kernel function is used for SVM training. The background training set consisted of 1744 conversation sides from 340 speakers. These data are also chosen from the same corpora as the UBM data, and two parts of data do not have overlap. In our experiments, the size of
SVM features are $36^{*} 2048$ for GSV features and 2048 for the proposed phonotactic features.

\subsection{Experimental Results}

When calculating the phonotactic features, only top $C$ largest scoring mixture components for each cepstral feature frame are used. We compare the results of the system with different value of $C$ in Table 1.

\begin{tabular}{ccc}
\hline top $C$ & EER $(\%)$ & minDCF \\
\hline total & $\mathbf{9 . 5 8}$ & $\mathbf{0 . 0 4 0 8}$ \\
top 50 & 9.32 & 0.0431 \\
top 100 & 9.22 & 0.0411 \\
top 150 & 9.17 & 0.0409 \\
top 200 & 9.12 & 0.0405 \\
top 250 & $\mathbf{9 . 0 4}$ & $\mathbf{0 . 0 4 0 3}$ \\
top 300 & 9.07 & 0.0405 \\
\hline
\end{tabular}

Table 1. Performance for proposed system with different values of $C$

From Table 1, we can see that the proposed system achieves the best performance when $C$ is tuned to be 250 . This improvement can brings gains of 5.6\% EER and $1.2 \%$ minDCF compared to the system using all the mixture components for calculating average posterior probabilities (total).

\begin{tabular}{ccc}
\hline system & EER (\%) & minDCF \\
\hline GMM (a) & 9.01 & 0.0436 \\
GSV (b) & 7.37 & 0.0354 \\
Proposed System (c) & 9.04 & 0.0403 \\
Baseline (a+b) & $\mathbf{7 . 0 6}$ & $\mathbf{0 . 0 3 5 3}$ \\
All-combination $(\mathrm{a}+\mathrm{b}+\mathrm{c})$ & $\mathbf{6 . 4 7}$ & $\mathbf{0 . 0 3 2 3}$ \\
\hline
\end{tabular}

Table 2. EER and minDCF for different systems

Figure 3 shows the detection error tradeoff (DET) curves for the various systems. We compare the proposed system with standard GMM-UBM and GSV-SVM systems. The exact values of EER and minDCF for different systems are shown in Table 2. It can be seen that the propose system produces comparable performance to the two systems. Better performance can be obtained when combining the proposed system with the two standard cepstral systems. The system combining all the three approaches can achieve $8.4 \%$ relative improvement in EER and $9.3 \%$ relative improvement in minDCF, respectively, compared to the system only combining two baselines $(a+b)$. 


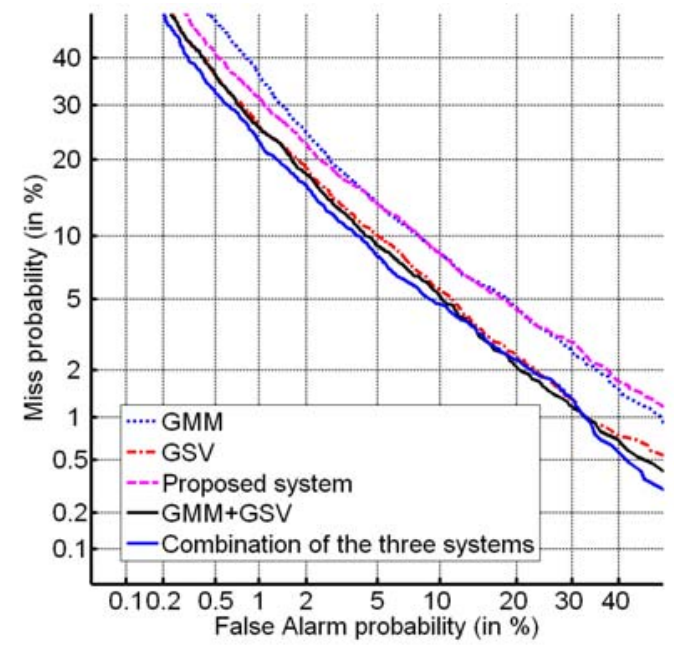

Fig. 3. DET curves for the proposed approach, GMM-UBM, GSV-SVM and various combinations

\section{CONCLUSIONS AND FUTURE WORK}

We have proposed a novel approach for speaker recognition. It uses a kind of phonotactic information as the feature for SVM modeling. This approach has been shown to have good performance on a NIST SRE 2006 task. Performance is found to be competitive with the commonly used GMM-UBM and GSV-SVM systems. Relative gains of $8.4 \%$ EER and 9.3\% minDCF are obtained when combining the proposed approach with the system combining the two baselines. The future work is focused on the application of the WCCN technique [13][14] in the proposed system to reduce the influence of the inter-session variability.

\section{REFERENCES}

[1] D.A. Reynolds, T.F. Quatieri, and R.B. Dunn, "Speaker Verification Using Adapted Gaussian Mixture Models," Digital Signal Processing, vol. 10, no. 1-3, pp. 19-41, 2000.

[2] N. Brummer, L. Burget, J. Cernocky, O. Glembek, F. Grezl, M. Karafiat, DA van Leeuwen, P. Matejka, P. Schwarz, and A. Strasheim, "Fusion of Heterogeneous Speaker Recognition Systems in the STBU Submission for the NIST Speaker Recognition Evaluation 2006," Audio, Speech and Language Processing, IEEE Transactions on, vol. 15, no. 7, pp. 2072-2084, 2007.

[3] W.M. Campbell, D.E. Sturim, and D.A. Reynolds, "Support Vector Machines Using GMM Supervectors for Speaker Verification," Signal Processing Letters, IEEE, vol. 13, no. 5, pp. 308-311, 2006.
[4] W.M. Campbell, D. Sturim, D.A. Reynolds, and A. Solomonoff, "SVM based speaker verification using a GMM supervector kernel and NAP variability compensation," Proc. ICASSP, pp. 97-100, 2006.

[5] A. Park and T.J. Hazen, "ASR Dependent Techniques for Speaker Identification," in Seventh International Conference on Spoken Language Processing. ISCA, 2002.

[6] D.E. Sturim, D.A. Reynolds, R.B. Dunn, and T.F. Quatieri, "Speaker verification using text-constrained Gaussian mixture models," Acoustics, Speech, and Signal Processing, 2002. Proceedings.(ICASSP'02). IEEE International Conference on, vol. 1, 2002.

[7] K. Boakye and B. Peskin, "Text-Constrained Speaker Recognition on a Text-Independent Task," in ODYSSEY04-The Speaker and Language Recognition Workshop. ISCA, 2004.

[8] D.A. Reynolds and R.C. Rose, "Robust textindependent speaker identification using Gaussian mixture speaker models," Speech and Audio Processing, IEEE Transactions on, vol. 3, no. 1, pp. 72-83, 1995.

[9] J.L. Gauvain and C.H. Lee, "Maximum a posteriori estimation for multivariate Gaussian mixtureobservations of Markov chains," Speech and Audio Processing, IEEE Transactions on, vol. 2, no. 2, pp. 291-298, 1994.

[10] P. Matejka, L. Burget, P. Schwarz, O. Glembek, M. Karafiat, F. Grezl, J. Cernocky, DA van Leeuwen, N. Brummer, and A. Strasheim, "STBU system for the NIST 2006 speaker recognition evaluation," Proc. ICASSP, 2007.

[11] "The NIST Year $2006 \quad$ Speaker Recognition Evaluation Plan," http://www.nist.gov/speech/tests/spk/2006/index.html, 2006.

[12] R. Collobert, S. Bengio, and R.C. Williamson, "SVMTorch: Support Vector Machines for Large-Scale Regression Problems," Journal of Machine Learning Research, vol. 1, no. 2, pp. 143-160, 2001.

[13] A.O. Hatch, S. Kajarekar, and A. Stolcke, "Within-Class Covariance Normalization for SVM-Based Speaker Recognition," in Ninth International Conference on Spoken Language Processing. ISCA, 2006.

[14] A. Stolcke, S.S. Kajarekar, L. Ferrer, and Shrinberg E., "Speaker Recognition With Session variability Normalization Based on MLLR Adaptation Transforms," IEEE Transactions On Audio, Speech, and Language Processing, vol. 15, no. 7, pp. 1987-1998, 2007. 